Europhysics Letters

PREPRINT

\title{
BCS-BEC crossover in an asymmetric two-component Fermi gas
}

\author{
XIA-Ji LiU ${ }^{1}$ and Hui Hu${ }^{1,2}$ \\ 1 ARC Centre of Excellence for Quantum-Atom Optics, Department of Physics, Uni- \\ versity of Queensland, Brisbane, Queensland 4072, Australia \\ 2 Department of Physics, Renmin University of China, Beijing 100872, China
}

\author{
PACS. 03.75.Hh - Static properties of condensates; thermodynamical, statistical and struc- \\ tural properties. \\ PACS. 03.75.Ss - Degenerate Fermi gases. \\ PACS. 05.30.Fk - Fermion systems and electron gas.
}

\begin{abstract}
We discuss the superfluid phase transition of a strongly interacting Fermi gas with unequal (asymmetric) chemical potentials in two pairing hyperfine states, and map out its phase diagram near the BCS-BEC crossover. Our approach includes the fluctuation contributions of "preformed Cooper pairs" to the thermodynamic potential at finite temperature. We show that, below a critical difference in chemical potentials between species, a normal gas is unstable towards the formation of either a finite-momentum paired Fulde-Ferrell-Larkin-Ovchinnikov superconducting phase or a uniform superfluid, depending on the asymmetry and interaction strengths. We determine the value of critical chemical potential mismatch, and find that it is consistent with a recent measurement by Zwierlein et al. [Science 311, 492 (2006)].
\end{abstract}

Recent experimental advances in manipulating ultracold atomic Fermi gases via a Feshbach resonance have attracted a great amount of interest in widely varying fields from condensed matter physics, atomic molecular and optical physics, to particle and astro physics [1-3]. Thanks to this precisely controllable environment, it is now possible to experimentally explore fundamental problems of many-body physics in the strongly interacting region. An example of particular interest is the understanding of fermionic pairings of two-component Fermi gases with mismatched Fermi surfaces or chemical potentials, in the crossover from the weak coupling Bardeen-Cooper-Schrieffer (BCS) regime to the strongly correlated BoseEinstein condensate (BEC) regime [4-12].

In standard BCS superconductivity, the presence of a Fermi surface mismatch suppresses superfluidity, and the pairing mechanism may be qualitatively altered. Various competing states have been proposed within mean-field theory to describe the ground state of an asymmetric Fermi gas in the weak coupling BCS regime: the Fulde-Ferrell-Larkin-Ovchinnikov (FFLO) state [4], where Cooper pairs possess a finite center-of-mass momentum, the breached pair phase [5] and the Sarma phase [6,7], or phase separation phases with a paired BCS superfluid being surrounded by an unpaired normal gas [8]. When the interaction strength is tuned across the BCS-BEC crossover, a rich phase diagram consisting of several these scenarios has

(C) EDP Sciences 
also been put forward [9-11]. Very recently, there have been two experimental investigations of fermionic superfluidity of ${ }^{6} \mathrm{Li}$ atoms with mismatched Fermi surfaces $[2,3]$.

In contrast to these earlier studies, in this paper we tackle the crossover problem of a homogeneous asymmetric Fermi gases at finite temperature, starting from a well-defined normal state. Our goal is to determine the superfluid transition temperature and to obtain a reliable phase diagram for these systems beyond mean-field. Our calculations reveal several notable features: (i) The normal state is stable above a critical chemical potential mismatch. The critical value predicted is in qualitative agreement with the recent measurement. (ii) On the BCS side, the critical value is roughly proportional to the transition temperature of a symmetric gas and, therefore is exponentially small. Below it, the normal gas is unstable towards the formation of FFLO states. As the mismatch of Fermi surfaces decreases, a uniform BCS superfluid is more favorable. (iii) In the strong coupling BEC regime, the critical difference in chemical potentials is of the order of binding energy. The superfluid state, well described by a mixture of tightly bounded Cooper pairs and unpaired fermions in this limit, is thereby remarkable robust. (iv) The transition temperatures at fixed chemical potential imbalances are also determined, giving rise to a finite-temperature phase diagram.

All these results are derived below by generalizing a thermodynamic approach by Nozières and Schmitt-Rink (NSR) [13-15] to asymmetric Fermi gases. This approach takes into account the large fluctuation effects that are necessary in order to capture the essential physics at the crossover.

NSR approach to an asymmetric Fermi gas. - Because the Feshbach resonance of ${ }^{6} \mathrm{Li}$ atoms used in the experiment is extremely broad $[16,17]$, we can use a single-channel model to describe the Fermi gas across a Feshbach resonance:

$$
\mathcal{H}=\sum_{\mathbf{k} \sigma}\left(\epsilon_{\mathbf{k}}-\mu_{\sigma}\right) c_{\mathbf{k} \sigma}^{+} c_{\mathbf{k} \sigma}+U \sum_{\mathbf{k} k^{\prime} \mathbf{q}} c_{\mathbf{k} \uparrow}^{+} c_{\mathbf{q}-\mathbf{k} \downarrow}^{+} c_{\mathbf{q}-\mathbf{k}^{\prime} \downarrow} c_{\mathbf{k}^{\prime} \uparrow}
$$

Here $c_{\mathbf{k} \sigma}^{+}$is the creation operator for the fermionic atoms, and the pseudospins $\sigma=\uparrow, \downarrow$ denote the two hyperfine states of ${ }^{6} \mathrm{Li}$. The masses are the same, so $\epsilon_{\mathbf{k}}=\hbar^{2} \mathbf{k}^{2} / 2 m$ for both species, but the chemical potentials are different, i.e., $\mu_{\uparrow, \downarrow}=\mu \pm \delta \mu$, to account for the asymmetry. We will focus on the situation with fixed total particle number $n=n_{\uparrow}+n_{\downarrow}$ and fixed chemical potential difference. $U$ is the effective interaction strength and is related to the $s$-wave scattering length $a$ via the regularization: $\left(4 \pi \hbar^{2} a / m\right)^{-1}=U^{-1}+\sum_{\mathbf{k}}\left(2 \epsilon_{\mathbf{k}}\right)^{-1}$. A two-body bound state arises in vacuum once the scattering length $a$ becomes positive.

Within the NSR approach [13], there are two essential ingredients in the thermodynamic potential at a temperature $T: \Omega(T, \mu, \delta \mu)=\Omega_{0}+\Omega_{p f}$. Here $\Omega_{0}$ corresponds to a free Fermi gas,

$$
\Omega_{0}=\frac{1}{\beta} \sum_{\mathbf{k}} \ln f\left[-\left(\epsilon_{\mathbf{k}}-\mu_{\uparrow}\right)\right]+\ln f\left[-\left(\epsilon_{\mathbf{k}}-\mu_{\downarrow}\right)\right],
$$

where $\beta=1 / k_{B} T$ and $f(x)=[\exp (\beta x)+1]^{-1}$ is the Fermi distribution function, while $\Omega_{p f}$ is associated with the pairing fluctuation contributions, and may be determined by summing an infinite series of ladder diagrams [13],

$$
\begin{aligned}
\Omega_{p f} & =\frac{1}{\beta} \sum_{\mathbf{q}, i \nu_{n}} \ln \left[-\Gamma^{-1}\left(\mathbf{q}, i \nu_{n}\right)\right] e^{i \nu_{n} 0^{+}}, \\
& =-\sum_{\mathbf{q}} \int_{-\infty}^{+\infty} \frac{d \omega}{\pi} g(\omega) \delta(\mathbf{q}, \omega) .
\end{aligned}
$$


Here $\mathbf{q}$ is the center-of-mass momentum, $i \nu_{n}=2 \pi n / \beta$ is the bosonic Matsubara frequency, $g(\omega)=[\exp (\beta \omega)-1]^{-1}$ is the Bose distribution function, and the particle-particle vertex function $\Gamma^{-1}\left(\mathbf{q}, i \nu_{n}\right)=1 / U+\chi_{\text {pair }}^{0}$ may be written as

$$
\Gamma^{-1}=\frac{m}{4 \pi \hbar^{2} a}+\sum_{\mathbf{k}}\left[\frac{f\left(\xi_{+}\right)+f\left(\xi_{-}\right)-1}{i \nu_{n}-2 \epsilon_{\mathbf{q} / \mathbf{2}}-2 \epsilon_{\mathbf{k}}+2 \mu}-\frac{1}{2 \epsilon_{\mathbf{k}}}\right]
$$

with $\xi_{ \pm}=\epsilon_{\mathbf{q} / 2 \pm \mathbf{k}}-\mu \mp \delta \mu$. Following NSR, in Eq. (4) we have converted the sum over $i \nu_{n}$ into a contour integral, and have rewritten $\Omega_{p f}$ in terms of a phase shift defined by $\delta(\mathbf{q}, \omega)=$ $-\operatorname{Im} \ln \left[-\Gamma^{-1}\left(\mathbf{q}, i \nu_{n} \rightarrow \omega+i 0^{+}\right)\right]$. The chemical potential $\mu$ for our model is determined from the number identity $n=-\partial \Omega / \partial \mu$, or,

$$
n=n_{F}^{0}(T, \mu, \delta \mu)+2 n_{B}(T, \mu, \delta \mu)
$$

where $n_{F}^{0}=\sum_{\mathbf{k} \sigma} f\left(\epsilon_{\mathbf{k}}-\mu_{\sigma}\right)$, and $n_{B}=-\partial \Omega_{p f} / \partial(2 \mu)=1 / \pi \sum_{\mathbf{q}} \int_{-\infty}^{+\infty} d \omega g(\omega) \partial \delta(\mathbf{q}, \omega) / \partial(2 \mu)$ may be interpreted as the number of "preformed Cooper pairs". The superfluid phase transition occurs when the particle-particle vertex function develops a pole at $i \nu_{n}=0$ for a certain value of $\mathbf{q}$. Therefore, the transition temperature $T_{c}$ can be conveniently obtained by the Thouless criterion,

$$
\left.\max \Gamma^{-1}\left(\mathbf{q}, i \nu_{n}=0\right)\right|_{T=T_{c}}=0,
$$

which is generalized here to take into account a nonzero center-of-mass momentum. We must solve Eq. (6) together with Eq.(17) self-consistently, to obtain $\mu\left(T_{c}\right)$ and $T_{c}$ for given chemical potential imbalance $\delta \mu$ and interaction coupling, where $k_{F}=\left(3 \pi^{2} n\right)^{1 / 3}$ is the Fermi wave vector.

$B C S$ and BEC limits. - The NSR formalism presented above is a simplified description of the full crossover problem for an asymmetric Fermi gas. It is asymptotically exact in the extreme weak or strong coupling limit $\left(1 / k_{F} a \rightarrow \pm \infty\right)$. In between, it is believed to provide a qualitative interpolation scheme [13].

In the weak coupling limit, the phase shift $\delta(\mathbf{q}, \omega)$ is small: the number equation (6) reduces to $n=n_{F}^{0}(T, \mu, \delta \mu)$. The chemical potential is therefore that of a non-interacting gas of fermions, $\mu \sim \epsilon_{F}=\hbar^{2} k_{F}^{2} / 2 m$. Keeping in mind that $\delta \mu, T_{c} \ll \mu$, to leading order of $1 /(\beta \mu)$ and $q v_{F} / \delta \mu$, one finds the following expression for the inverse of two-particle vertex function:

$$
\frac{2 \pi^{2} \hbar^{2}}{m k_{F}} \Gamma^{-1}=\left[\ln \frac{T}{T_{c}^{0}}+h_{1}(\delta \mu)\right]-\frac{\epsilon_{F}}{6} \frac{\hbar^{2} q^{2}}{2 m} h_{2}(\delta \mu),
$$

where $k_{B} T_{c}^{0}=k_{B} T_{B C S}=(8 / \pi) e^{\gamma-2} \epsilon_{F} \exp \left(\pi / 2 k_{F} a\right)$ is the BCS transition temperature for a respective symmetric gas, $h_{1}(x)=-\int_{0}^{\infty} d y \ln (\beta y)\left[f^{\prime}(y+x)+f^{\prime}(y-x)\right]+\gamma+\ln (4 / \pi)$ and $h_{2}(x)=-\int_{0}^{\infty} d y(1 / y)\left[f^{\prime \prime}(y+x)+f^{\prime \prime}(y-x)\right]$. At small chemical potential imbalance $\left(\delta \mu / T_{c}^{0} \rightarrow 0\right), h_{2}(\delta \mu)>0$, and the superfluid instability occurs at $\mathbf{q}=0$. Asymptotically, we obtain

$$
\frac{T_{c}(\delta \mu)}{T_{c}^{0}}=\left[1-\frac{7}{12}\left(8 \zeta^{\prime}(-2)-\frac{\zeta(3)}{\pi^{2}}\right)\left(\frac{\delta \mu}{k_{B} T_{c}^{0}}\right)^{2}\right],
$$

where $\zeta(x)$ is the Riemann zeta function. As $\delta \mu$ increases, $h_{2}(\delta \mu)$ crosses zero and then becomes negative. Hence the maximum value of $\Gamma^{-1}$ locates at a nonzero center-of-mass momentum, triggering the emergence of a non-uniform FFLO state. The condition $h_{2}(\delta \mu)=0$ defines a tricritical point connecting both two superfluid phases and the normal phase. We find numerically that $\delta \mu_{t r i} \simeq 1.07 k_{B} T_{c}^{0}$ and $T_{c, t r i} \simeq 0.56 T_{c}^{0}$, in agreement with a previous 
study from Ginzburg-Landau theory [18]. As $\delta \mu$ increases further and approaches a critical imbalance, the transition temperature shrinks to zero, marking a quantum phase transition to the normal state. In this case the expression (8) becomes inapplicable, since the resulting $q v_{F}$ may become comparable with $\delta \mu$. In the limit of $\beta \rightarrow \infty$, alternatively we may cast Eq. (15) in the form,

$$
\frac{2 \pi^{2} \hbar^{2}}{m k_{F}} \Gamma^{-1}=\left[\ln \frac{k_{B} T_{c}^{0}}{\delta \mu}+F(x)\right]+\frac{\pi^{2}}{6}\left(\frac{k_{B} T}{\delta \mu}\right)^{2} \frac{1}{1-x^{2}}
$$

where $F(x)=1-\gamma-\ln (2 / \pi)-1 / 2 \ln \left|x^{2}-1\right|-1 /(2 x) \ln |(x+1) /(x-1)|$ and $x=q v_{F} / \delta \mu$. The function $F(x)$ has a maximum at $x=x_{c} \simeq 1.20$, with $F\left(x_{c}\right) \simeq 0.286$. Thus the quantum phase transition takes place at $\delta \mu_{c} \simeq 1.331 k_{B} T_{c}^{0}$. Slightly below $\delta \mu_{c}$ the transition temperature shows a square root behavior,

$$
\frac{k_{B} T_{c}(\delta \mu)}{\delta \mu_{c}}=\frac{\sqrt{6\left(x_{c}^{2}-1\right)}}{\pi}\left(\frac{\delta \mu_{c}-\delta \mu}{\delta \mu_{c}}\right)^{1 / 2} .
$$

Deep within the BEC regime, the results are greatly affected by the phase shift contributions. The particle-particle vertex function develops a discrete pole at a positive frequency $\omega=\epsilon_{q} / 2-2 \mu+\epsilon_{b}$, where $\epsilon_{b}=\hbar^{2} / m a^{2}$ is the binding energy. Thus, all fermions in the less populated hyperfine state will pair up with atoms in other state to form bound pairs. As a result, in the low energy regime the phase shift is dominated by a bound state part, i.e., $\delta(q, \omega) \simeq \pi \theta\left(\epsilon_{q} / 2-2 \mu+\epsilon_{b}\right)$, and the number of pairs consequently acquires a standard form of an ideal Bose gas: $n_{B}=n_{\downarrow} \simeq \sum_{\mathbf{q}} g\left(\epsilon_{\mathbf{q}} / 2-2 \mu+\epsilon_{b}\right)$. Accordingly the chemical potential $\mu$ asymptotes to half the binding energy. On the other hand, to accommodate the remaining free excess fermions with density $\delta n=n_{\uparrow}-n_{\downarrow}>0$, the chemical potential imbalance $\delta \mu$ should be necessary large. One may anticipate that $\mu_{\downarrow} \sim-\epsilon_{b}$ while $\mu_{\uparrow} \sim \epsilon_{F}(\delta n)$, where $\epsilon_{F}(\delta n)$ is the respective Fermi level of these excess fermions: $\delta n \approx \sum_{\mathbf{k}} f\left(\epsilon_{\mathbf{k}}-\mu-\delta \mu\right)$. At the transition point the Thouless criterion gives rise to $\mu\left(T_{c}\right)=-\epsilon_{b} / 2$. The superfluid transition temperature is therefore determined by the strong coupling number equation describing a mixture of non-interacting bosons and fermions,

$$
n=\sum_{\mathbf{k}} \frac{1}{e^{\left(\epsilon_{\mathbf{k}}-\mu_{\uparrow}\right) / k_{B} T_{c}}+1}+2 \zeta\left(\frac{3}{2}\right)\left[\frac{m k_{B} T_{c}}{\pi \hbar^{2}}\right]^{3 / 2} .
$$

By setting $T_{c}=0$, we obtain a critical chemical potential imbalance: $\delta \mu_{c}=\epsilon_{b} / 2+2^{2 / 3} \epsilon_{F}$.

Two striking features emerge from this analysis: first, in both BCS and BEC-like phases, the superfluid state is destroyed by a sufficient large chemical potential imbalance. On the BCS side, the critical difference in chemical potentials is exponentially small, while deep within the BEC regime, it is set by the binding energy. Second, in the BCS-like phase, the preformed Cooper pairs can adjust its center-of-mass momentum in response to the Fermi surface mismatch. This results an inhomogeneous FFLO superfluid state in the vicinity of critical chemical potential difference. With these background, let us turn to the numerical NSR calculations.

Transition temperatures and phase diagrams.-Figure 1 presents our results for the superfluid transition temperature as a function of the chemical potential imbalance at various interaction strengths. The dashed lines shows the region with the onset of FFLO phases. We plot the number of "preformed Cooper pairs" in dotted lines. Two mechanisms for the depression of transition temperatures with increasing imbalance may be identified. On the 

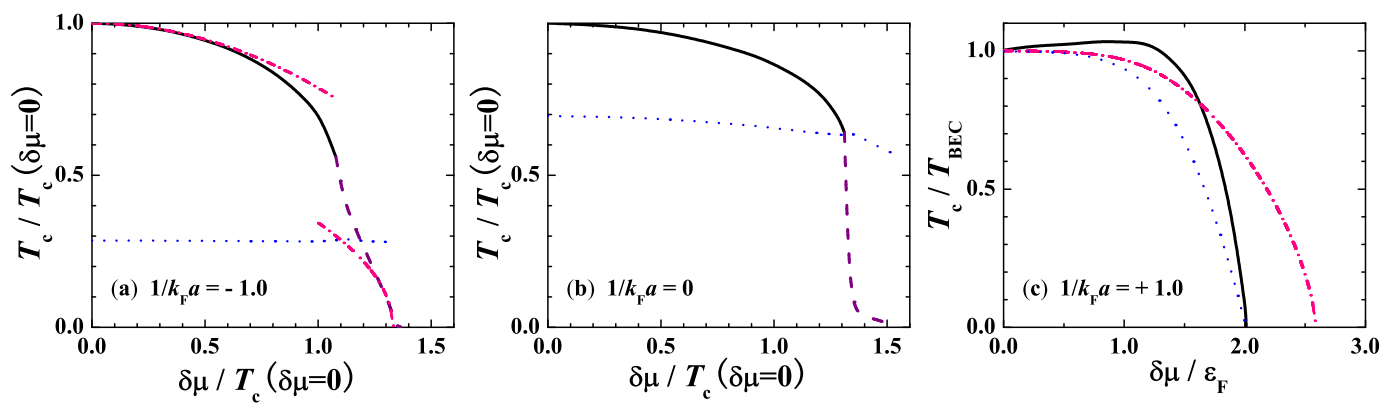

Fig. 1 - (color online) Superfluid transition temperature $T_{c}$ as a function of the chemical potential imbalance at various interaction strengths: (a) $1 / k_{F} a=-1$, (b) the unitary limit $1 / k_{F} a=0$, and (c) $1 / k_{F} a=+1$. The phase transitions into an inhomogeneous FFLO state have been marked by dashed lines. The dot-dashed lines in (a) shows, respectively, the asymptotic behavior near $\delta \mu=0$ and $\delta \mu=\delta \mu_{c}$ in Eqs. (9) and (11), while that in (c) shows the $T_{c}$ determined from the strong coupling number equation (12). The dotted lines display the number of "preformed Cooper pairs". In (c) $T_{B E C}=\left(\hbar^{2} / m\right) \pi[n /(2 \varsigma(3 / 2))]^{2 / 3} / k_{B}$ is the transition temperature for an ideal Bose gas.

strong coupling regime, the decrease of $T_{c}$ is accompanied with the reduction of number of tightly bounded pairs, as expected from the general picture of BEC. In contrast, in the opposite BCS-like phase, the number of pairs keeps almost constant. These pairs, resulting from the many-body effects in this case, would be very fragile with respect to the Pauli blocking effects arising from mismatched Fermi surfaces. We therefore explain the reduction of $T_{c}$ as due to the loss of phase coherence between pairs.

Figure 2a gives the critical chemical potential imbalance $\delta \mu_{c}$ throughout the BCS-BEC crossover, constituting a zero-temperature phase diagram. For comparison, we plot the experimental data on the critical Fermi energy difference $\delta E_{F} / \epsilon_{F}$. These data are obtained indirectly by assuming a non-interacting dispersion: $\delta E_{F} / \epsilon_{F}=\left[\left(1+\delta_{c}\right)^{1 / 3}-\left(1-\delta_{c}\right)^{1 / 3}\right] / 2$, where $\delta_{c}$ is the measured critical population imbalance (see, i.e., the Fig. 5 in Ref. [2]). We find that our predictions agree qualitatively with the experimental results in the BCS-unitarity regime. However, on the BEC side they are not consistent. To understand this discrepancy, several remarks should be in order: First, our calculation is for a homogeneous gas, while the experiment is done in a trap. The presence of the trap tends to yield a phase separation, which may further complicate the comparison. Secondly, the experiment is performed at a unknown temperature. A close examination of the experimental data in the unitary limit suggests that there is an appreciable effect due to the finite temperature. Finally, the experimental data refer to the critical Fermi energy difference, instead of the critical chemical potential difference as we calculated. Only in the weakly coupling BCS regime do the chemical potentials equal the Fermi energies. Thus it appears to be the most serious reason responsible for the discrepancy between our predictions and experimental data on the BEC side. In this regard, a theoretical calculation for the critical population imbalance will be useful, enabling a direct comparison with the experiment. Unfortunately, in a region around the unitary limit $\left(-0.5<1 / k_{F} a<+0.2\right.$, see Fig. $\left.2 \mathrm{~b}\right)$, we find that the NSR approach generally leads to a negative population imbalance at a positive chemical potential difference, implying an un-physical compressibility, i.e., $\partial \delta n / \partial \delta \mu<0$. This suggests the breakdown of the NSR treatment around the unitarity regime. We note that the negative compressibility is in close connection with the non-monotonous behaviour of the transition temperature predicted by NSR scheme for a symmetric Fermi gas $[13,14]$. Presumably, the breakdown of NSR approach is due to the 

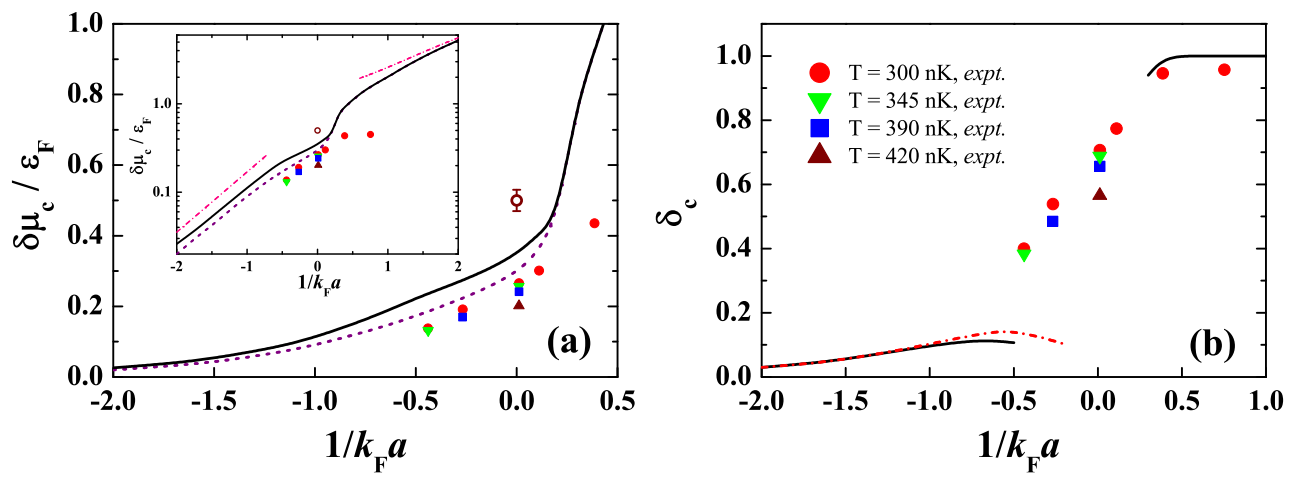

Fig. 2 - (color online) (a) Predicted critical chemical potential imbalance (solid line) in the BCSBEC crossover. Solid symbols are the experimental data on the critical Fermi energy difference. The open circle with an error bar in the unitary is a rough estimate from quantum Monte Carlo simulations in Ref. [12]. The position of the tricritical points $\left(\delta \mu_{t r i}\right)$ has been plotted by a dashed line. Inset shows the critical imbalance in the logarithmic scale, where two dotted-dashed lines represent, respectively, the critical imbalance in the extreme BCS and BEC limits, i.e., $\delta \mu_{c} \simeq 1.331 k_{B} T_{B C S}$ and $\delta \mu_{c}=\epsilon_{b} / 2+2^{2 / 3} \epsilon_{F}$. (b) Predicted critical population imbalance $\delta_{c}$. The NSR approach (at $\left.T=0\right)$ breaks down around the unitarity regime $-0.5<1 / k_{F} a<+0.2$, and thereby $\delta_{c}$ is unknown. The dotted-dashed line shows the perturbation result in the BCS limit: $\delta_{c}=3 /\left[2\left(1-2 k_{F} a / \pi\right)\right] \delta \mu_{c}$.

exclusion of the interactions between Cooper pairs, and may be avoided by a self-consistent improvement, with which the un-physical peak structure of $T_{c}$ is shown to be removed $[17,19]$. In Fig. 2b, we report the critical population imbalance outside of the unitarity regime. The two experimental points on the BEC side now become consistent with our theory.

In Fig. 2a we have also shown the chemical potential at the tricritical point (with nonzero $T_{c}$ ) by a dashed line. Therefore, the area enclosed by the solid line and dashed line marks the possible region for forming the FFLO state. The region ends up around the unitary limit. This fact, together with the low $T_{c}$ for FFLO phases, may be used to understand the reason of why such states are not observed in the experiment.

For completeness, in Fig. 3 we consider the transition temperature against the interaction strength at several fixed values of chemical potential imbalance, which makes up a finite temperature phase diagram. In particular, there is no superfluid state on the BCS side above $\delta \mu \simeq 0.3 \epsilon_{F}$.

Conclusions. - In summary, based on a generalized NSR approximation beyond meanfield, we have qualitatively determined the superfluid transition temperature and a phase diagram for a two-component Fermi gas near a Feshbach resonance with mismatched Fermi surfaces. The resulting critical imbalance in chemical potentials agrees qualitatively well with the recent experimental findings [2]. While our approach starts from a well-defined normal state and thereby avoids the complication of assuming a possible pairing scheme, it cannot be used to identify the transition between different competing phases within the superfluid domain. This fascinating issue could be addressed by extending the current NSR approach to the broken-symmetry state [20].

We acknowledge stimulating discussions with Professor P. D. Drummond. This work was supported by the Australian Research Council Center of Excellence and the National Science 


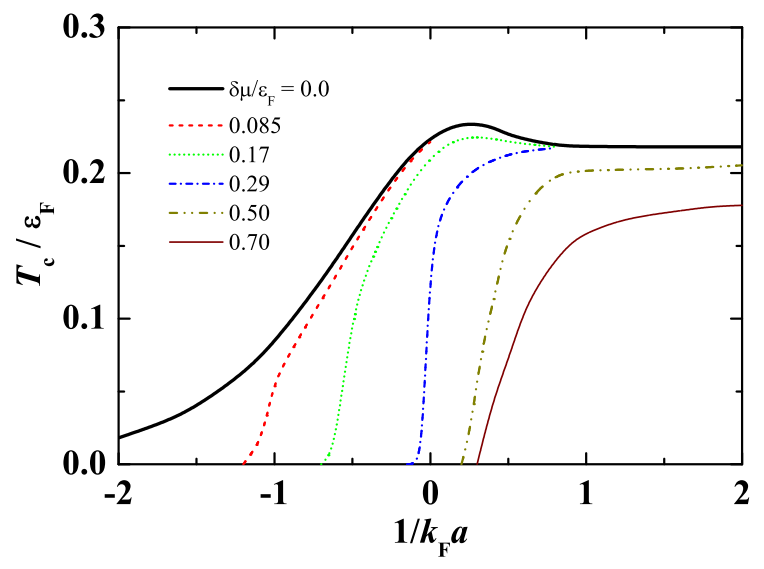

Fig. 3 - (color online) Finite-temperature phase diagram with the superfluid transition temperature at the crossover plotted versus chemical potential imbalances. For a better illustration, we have subtracted half the binding energy from the imbalance, except for the curve with $\delta \mu=0$.

Foundation of China with Grant No. 10574080.

\section{REFERENCES}

[1] Kinast J. et al., Phys. Rev. Lett., 92 (2004) 150402; Bartenstein M. et al., Phys. Rev. Lett., 92 (2004) 203201; Chin C. et al., Science, 305 (2004) 1128; Kinast J. et al., Science, 307 (2005) 1296; Zwierlein M. W. et al., Nature, 435 (2005) 1047; Hu H. et al., Phys. Rev. Lett., 93 (2004) 190403.

[2] Zwierlein M. W. et al., Science, 311 (2006) 492.

[3] Partridge G. B. et al., Science, 311 (2006) 503.

[4] Fulde P. and Ferrell R. A., Phys. Rev., 135 (1964) A550; Larkin A. I. and Ovchinnikov Y. N., Zh. Eksp. Teor. Fiz., 47 (1964) 1136[Sov. Phys. JETP, 20 (1965) 762].

[5] Liu W. V. and Wilczek F., Phys. Rev. Lett., 90 (2003) 047002.

[6] Sarma G., J. Phys. Chem. Solids, 24 (1963) 1029.

[7] Pao C.-H., Wu S.-T, and YiP S.-K., Phys. Rev. B, 73 (2006) 132506.

[8] Bedaque P. F., Caldas H., and Rupak G., Phys. Rev. Lett., 91 (2003) 247002; Chevy F., Phys. Rev. Lett., 96 (2006) 130401; Haque M. and Stoof H. T. C., cond-mat/0601321, preprint, 2006.

[9] Son D. T. and Stephanov M. A., cond-mat/0507586, preprint, 2005.

[10] Sheehy D. E. and Radzihovsky L., Phys. Rev. Lett., 96 (2006) 060401.

[11] Hu H. and Liu X.-J., Phys. Rev. A, 73 (2006) 051603(R).

[12] Carlson J. and Reddy S., Phys. Rev. Lett., 95 (2005) 060401.

[13] Nozières P. and Schmitt-Rink S., J. Low Temp. Phys., 59 (1985) 195;

[14] Sá de Melo C. A. R., Randeria M., and J. R. Engelbrecht J. R., Phys. Rev. Lett., 71 (1993) 3202.

[15] Ohashi Y. and Griffin A., Phys. Rev. Lett., 89 (2002) 130402.

[16] Partridge G. B. et al., Phys. Rev. Lett., 95 (2005) 020404.

[17] Liu X.-J. and Hu H., Phys. Rev. A, 72 (2005) 063613.

[18] Casalbuoni R. and Nardulli G., Rev. Mod. Phys., 76 (2004) 263.

[19] Haussmann R., Phys. Rev. B, 49 (1994) 12975.

[20] Hu H., Liu X.-J., and Drummond P. D., Europhys. Lett., 74 (2006) 574; Hu H., Liu X.-J., and Drummond P. D., Phys. Rev. A, 73 (2006) 023617. 